\title{
- La Tasa de Descuento en la Evaluación de Proyectos Y Negocios Empresariales
}

\author{
(a) Hér cul es Mol ina A \\ (2) Javier Del Carpio G.
}

\section{RESUMEN}

El artículo presenta los principales criterios para el uso de la tasa de descuento en los proyectos de inversión y planes de negocios, a partir del análisis y comparación de los problemas derivados en mercados de capitales emergentes e incompletos, utilizando el modelo clásico de asignación de precios de los activos de capital (CAPM), a fin de obtener resultados con mayor precisión en la estimación de los parámetros.

Palabras Claves: Tasa de descuento. Costo de oportunidad del capital. Flujo de caja. Rendimiento y riesgo.

Discount Rate In the ENTERPRISE Businesses AND PROJ ECTS ASSESSMENT ABSTRACT

This article presents the major criteria for using a discount rate in business plans and investment projects, out of the analysis and comparison of the problems arising in emerging and incomplete capital markets, by means of using the Capital Assets Price Assignment Classic Model (CAPM), with the aim of getting more accurate results as for parameters appraisal.

Key Words: Discount rate. Capital opportunity cost. Cash flow. Risk and yield.

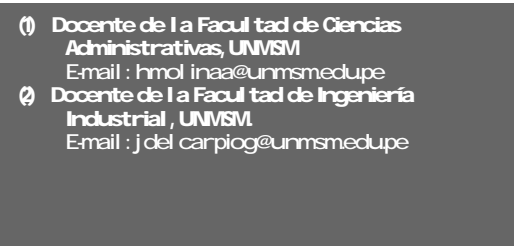

\section{INTRODUCCIÓN}

En el mundo de la empresa es de suma importancia tomar buenas decisiones de inversión. Pero frecuentemente se observan excelentes ideas en los proyectos y planes de negocios que en la práctica se ven deslucidos por un mal cálculo del valor presente neto, debido básicamente a una aplicación inadecuada de fórmulas en la estimación de la tasa de descuento o costo de capital que dejan de lado algunos conceptos básicos.

Una de las más importantes tareas de la evaluación y gestión de inversiones empresariales es estimar el costo de oportunidad de su capital. En la teoría moderna, la toma de decisiones en incertidumbre introduce un marco conceptual para estimar el riesgo y el rendimiento de un activo que es parte de una cartera o portafolio y bajo condiciones de equilibrio de mercado. Este marco conceptual se denomina modelo de asignación de precios de los activos de capital o CAPM (Capital Asset Pricing Model). En este modelo el riesgo de una inversión se divide en riesgo sistemático o riesgo de mercado(no diversificable) y riesgo no sistemático (diversificable) o riesgo específico de una empresa.

El primer tipo de riesgo es el más importante para el CAPM y está medido por su coeficiente beta. Este coeficiente relaciona el exceso de rendimiento de la acción respecto de la tasa libre de riesgo y el exceso de rendimiento de mercado respecto a la tasa libre de riesgo. El riesgo diversificable o riesgo no sistemático surge por aspectos como litigios, huelgas, programas de comercialización con o sin éxito y otros eventos que son únicos para una empresa en particular. Puesto que estos eventos son esencialmente individuales, sus efectos sobre una cartera de inversión pueden ser controlados y eliminados mediante la diversificación.

La forma tradicional de obtener el coeficiente beta es por medio de una regresión lineal de dos variables según el supuesto de que el rendimiento en exceso de la inversión, analizada como una serie de tiempo, tiene varianza condicional homoscedástica.

El modelo CAPM, como otros modelos que miden el rendimiento esperado y el riesgo de un activo, han sido severamente criticados por una serie de autores, los cuales encuentran inadecuada la estructura de estos modelos para estimar y predecir el precio al riesgo. Algunas de estas críticas se basan en que la varianza de largo plazo se supone constante. Otras críticas se sustentan con pruebas de regresión, las cuales muestran que las predicciones sobre la tasa del premio al riesgo medido por medio de las variables que se utilizan como explicativas son ineficientes. Existen críticas más fuertes que cuestionan la lógica empírica de los modelos de Harry Markowitz y William Sharpe, quienes propusieron el modelo CAPM. 
A pesar que el modelo CAPM y otros modelos, como el de valoración por arbitraje, que miden el riesgo de un activo han sido objeto de severas críticas, este sigue siendo muy útil en la evaluación de las inversiones empresariales.

En los últimos años, se pueden encontrar otra clase de modelos, pertenecientes a la teoría de series de tiempo. Esta clase de modelos son los llamados modelos de heteroscedasticidad condicional autorregresiva (ARCH, GARCH y ARCH-M), que tratan de superar las ineficiencias estructurales de los modelos financieros. Por otro lado, se considera que el mercado de capitales es eficiente sólo parcialmente. Hay tres dimensiones en la medición de la eficiencia: operacional, distributiva y de precios. Hay eficiencia operacional si todas las transacciones pueden realizarse transparentemente al mínimo costo posible. Hay eficiencia distributiva si cualquier activo financiero de igual riesgo ofrece la misma rentabilidad. Hay eficiencia en precios si toda la información disponible se refleja en los precios. A la vez hay tres grados de eficiencia en precios: débil, semifuerte y fuerte. En el grado débil los precios actuales reflejan al menos toda la información pasada. En el grado semifuerte, además de lo anterior, debe reflejar toda la información hecha pública a través de los estados financieros. En el grado fuerte, además de lo anterior, debe reflejar la información privada; por ejemplo, los planes de crecimiento de una empresa.

En este trabajo se aborda tres aspectos. Primero, se presenta el marco teórico para la estimación del riesgo empresarial lo que ayudará a definir la evaluación privada o financiera de las inversiones. Segundo, se muestra los típicos errores en la valoración. Tercero, se dan a conocer algunos datos de la evidencia empírica en la Bolsa de Valores de Lima para determinar la tasa de descuento a partir del precio de las acciones. No se tomarán en cuenta los efectos de la inflación y devaluación.

\section{MARCO CONCEPTUAL}

\section{E Model o de Val uación de Activos -CAPM}

El CAPM es un modelo de equilibrio general que se emplea para determinar la relación existente entre la rentabilidad y el riesgo de un portafolio o un título de inversión cuando el mercado de capitales se encuentra en equilibrio. El modelo asume, entre otras cosas, que todos los inversionistas en el mercado determinan el portafolio óptimo empleando el enfoque de Harry Markowitz.

El modelo CAPM tiene un planteamiento simple, y se sustenta en una serie de supuestos sobre el mer- cado de capitales. A pesar de que los supuestos del modelo no necesariamente se cumplen en la vida real, la capacidad predictiva del modelo ha demostrado ser efectiva.

\section{Supuestos del Model o CAPM}

Los supuestos del CAPM describen una situación extrema. El modelo se basa en que el mercado de capitales es perfecto, y no existe ningún tipo de restricción que impidan la participación de los inversionistas.

Este modelo se desarrolla en un mundo hipotético donde se hacen los siguientes supuestos acerca de los inversionistas y del conjunto de oportunidades de una cartera de inversión:

1. Los inversionistas son individuos que tienen aversión al riesgo y buscan maximizar la utilidad esperada de su riqueza al final del periodo. Los inversionistas evalúan los portafolios tomando en cuenta los retornos esperados y la desviación estándar de los diversos portafolios. Dados dos portafolios iguales, se escogerá aquel de menor desviación estándar.

2. Los inversionistas son tomadores de precios y poseen expectativas homogéneas acerca de los rendimientos de los activos, los cuales tienen una distribución normal conjunta.

3. Existe un activo libre de riesgo tal que los inversionistas pueden pedir en préstamo o prestar montos ilimitados a la tasa libre de riesgo. La tasa libre de riesgo es la misma para todos los inversionistas

4. Los valores son infinitamente divisibles. Las cantidades de todos los activos son negociables y perfectamente divisibles. Si un inversionista lo desea puede adquirir la fracción de una acción.

5. Existe información perfecta. Los mercados de activos están libres de fricciones; la información no tiene costo alguno y está al alcance de todos los inversionistas.

6. No existen imperfecciones en el mercado (como impuestos, leyes, etcétera). Es decir, los impuestos y los costos de transacción son irrelevantes.

Estos supuestos muestran que el CAPM se basa en los postulados de la teoría microeconómica, en donde el consumidor (el inversionista con aversión al riesgo) elige entre curvas de indiferencia que le proporcionan la misma utilidad entre el riesgo y el rendimiento. Esta elección entre el riesgo y el rendimiento lleva al inversionista, por un lado, a la formación de carteras y a la búsqueda de portafolios que incluyan, además de los activos riesgosos, valores cuya tasa es libre de riesgo, y por otro lado a enfrentarse a un mercado de fondos prestables que debe estar en equi- 
librio en cada momento del tiempo. Adicionalmente, como todo consumidor racional, el inversionista adverso al riesgo buscará máximizar el rendimiento esperado sobre sus activos y minimizar el riesgo. Esta conducta de los inversionistas hace que exista un conjunto de portafolios únicos que maximizan el rendimiento esperado de un activo y minimizan el riesgo; a esta serie de carteras se les denomina comúnmente portafolios eficientes.

\section{LA EVALUACIÓN PRIVADA O FINANCIERA}

En esta evaluación se debe incluir la interpretación objetiva de datos y buen criterio cualitativo. Básicamente, esta evaluación se realiza por medio del valor presente neto o VPN. Este criterio consiste en descontar los flujos de caja a una tasa de descuento que refleje el rendimiento de una alternativa de inversión similar al riesgo del proyecto o negocio. Pero ¿cuál es la tasa de descuento más apropiada para descontar los flujos de caja que ofrece un determinado proyecto o negocio?. Dar respuesta a esta pregunta requiere estudiar la estructura óptima de financiamiento o relación más conveniente que puede establecerse entre los recursos prestados(deuda) y el capital propio (D/C). A partir de los trabajos de Modigliani y Miller se plantea la proposición básica siguiente:

La capacidad de endeudamiento permite aumentar el rendimiento de una actividad empresarial debido al ahorro fiscal o escudo tributario generado por el pago de intereses de la deuda contraida. Esto confirma lo que se plantea teóricamente: en cualquier sistema de mercado donde los impuestos son diferentes de cero y donde los activos de inversión tienen diferentes riesgos, la estructura de capital tiene efecto sobre el valor de la ganancia total del inversionista en un proyecto o negocio empresarial.

Por otro lado, el costo de oportunidad de capital o COK es un concepto que expresa el rendimiento alternativo de igual riesgo económico. Pero cuando una parte del proyecto es financiado a través de préstamo o deuda, este COK se debe corregir para obtener otro apalancado que incluye efecto de la deuda. El valor de la prima de riesgo esperada para una inversión está directamente relacionado con la volatilidad de los rendimientos(b) que muestra esa inversión en relación con los rendimientos que ofrece el mercado de capitales. La tasa de descuento tiene dos componentes básicos: la tasa libre de riesgo(Rf), que toma en cuenta el valor del dinero en el tiempo y una prima por riesgo que ha de compensar el riesgo adicional de la inversión El costo promedio ponderado de capital o WACC(weight average capital cost), se define como costo promedio de las diferentes fuentes de financiamiento. Estas tasas, según la teoría del CAPM, se expresan del modo siguiente:

El rendimiento de una inversión(Ri) sería: $R$ inversión $=\mathrm{Ri}=\mathrm{Rf}+$ prima por riesgo de la inversión. Asimismo, el rendimiento para una cartera de inversión y para el mercado en su conjunto sería: Rmercado = $\mathrm{Rm}=\mathrm{Rf}+$ prima de riesgo de mercado .

Para hallar el rendimiento requerido de una inversión en particular es preciso relacionar el rendimiento de una inversión con el rendimiento del mercado o concretamente con la prima del mercado. Tal relación se puede establecer a través de la correlación de los excesos de retorno de la inversión o prima de riesgo de la inversión(Ri - Rf) en función de los excesos de retorno del mercado o prima de riesgo del mercado $(R m-R f)$, es decir, Rinversión = Rf + B.Rmercado.. Ese tipo de relación expresa la volatilidad de los rendimientos de la inversión y se denomina beta(Bi). Para hallar el beta hay que estimar una regresión lineal de dos variables usando el método de mínimos cuadrados ordinarios(MCO), donde la inclinación o pendiente(ß) de la línea de regresión establecerá la relación que hay entre la prima de la inversión y la de mercado (veáse Figura 1). El beta se puede interpretar como el grado de respuesta de la variabilidad de los rendimientos de la inversión a la variabilidad de los rendimientos del mercado. $\mathrm{Si} \mathrm{Bi}>1$, entonces tenemos que las variaciones en los rendimientos de la inversión serán mayores a las variaciones del rendimiento del mercado (inversiones riesgosas). $\mathrm{Si} \mathrm{Bi}=1$, el rendimiento de la inversión variará en la misma proporción que la variación del rendimiento de mercado (inversiones de riesgo promedio). Si $ß i<1$, entonces el valor de la inversión será menos riesgosa que el rendimiento del mercado (inversiones defensivas). Si ßi es negativo, se tendrá las llamadas inversiones superdefensivas (véase Figura 1).

De lo anterior se deduce que:

$\mathrm{Bi}=$ Prima de riesgo de la inversión/ Prima de riesgo de mercado $=(\mathrm{Ri}-\mathrm{Rf}) /(\mathrm{Rm}-\mathrm{Rf})$

Es decir: $(C O K-R f)=b .(R m-R f)$, de modo que:

$\mathrm{COK}=\mathrm{Rf}+\mathrm{b}(\mathrm{Rm}-\mathrm{Rf})+$ ? $\mathrm{t}$, que es la conocida expresión del CAPM

COKApal $=\mathrm{COK}+(1-\mathrm{t})(\mathrm{COK}-\mathrm{i}) \mathrm{D} / \mathrm{C}$

WACC $=\mathrm{i}(1-\mathrm{t}) \mathrm{D} /(\mathrm{C}+\mathrm{D})+\mathrm{COKApalC} /(\mathrm{C}+\mathrm{D})$

COK es el costo de oportunidad del capital o tasa de rendimiento mínima de la inversión.

COK Apal es el costo de oportunidad del capital apalancado. 
WACC es el costo promedio ponderado del capital. $\mathrm{Rf}$ es la tasa libre de riesgo = intercepto de la regresión o rendimiento autónomo.

Rm es el rendimiento del mercado.

$B$ es el beta, mide la volatilidad de los rendimientos relativos del proyecto. Coeficiente que mide el grado de riesgo del activo con respecto al rendimiento de mercado.

i es el costo de la deuda o préstamo de la empresa. $t$ es la tasa marginal de impuestos.

$\mathrm{D}$ es el monto de la deuda o del préstamo a valores de mercado.

C es el monto de capital a valores de mercado.

? t es el término de error aleatorio de la regresión en el periodo $t$.

Desde el punto de vista privado, las empresas pueden elaborar dos clases de flujos de caja: los económicos (inversión y los flujos del proyecto o giro del negocio, incluyendo la liquidación) y los financieros que comprenden los flujos económicos antes mencionados más: el desembolso del principal, las amortizaciones, pago de intereses del préstamo y el escudo tributario o escudo fiscal.

La evaluación financiera intenta valorar las ganancias generadas por el proyecto o por el negocio, y las que se derivan del financiamiento (i.e., el escudo tributario por los intereses cuando se tiene acceso al crédito comercial o subsidiado). Una sólida evaluación de proyectos que combina flujos debe utilizar tasas de descuento pertinente en cada caso.

Indicador es de I a Eval uación Privada La evaluación privada incluye cualquiera de los tres indicadores siguientes:

1. Ganancia económica del proyecto (GEP). Medido por el VPN económico, que se obtiene al descontar el flujo de caja económico a la tasa de descuento o costo de oportunidad del capital propio (COK sin apalancamiento).

2. Ganancias derivadas de la financiación. La ganancia por acceso al préstamo (GA) que se origina por tener acceso a un crédito a una tasa inferior a la de mercado. Y las ganancias por ahorro fiscal(GAF) o ganancias por escudo tributario(GET) de los intereses del financiamiento, que son aquellas que se derivan de un menor pago de impuestos al deducirse los intereses de la base imponible.

3. Ganancia total del inversionista (GTI). También llamado VPN total, VPNA(valor presente neto ajustado por financiamiento) o simplemente VPN financiero. En este caso puede haber tres alternativas; el primero suma el VPN económico y las dos ganancias derivadas del financiamiento (GA y GAF); en el segundo, se descuentan los flujos económicos al costo promedio ponderado del capital (WACC); y en la tercera, se descuentan los flujos financieros al COK apalancado. Los tres enfoques ofrecerán los mismos resultados.

Es importante tener en cuenta cómo se interpretan estos indicadores y cuáles son sus limitaciones.

EI VPN económico mide el valor intrínseco del negocio o del proyecto, es decir, su capacidad de generar valor agregado para las empresas. Para muchos es el indicador más importante porque da una idea del verdadero valor.

La ganancia por acceso al crédito se deriva de un subsidio, por el hecho de acceder al financiamiento a una tasa más barata. El escudo fiscal proviene de una norma que permite la deducción del gasto por intereses sobre la utilidad neta. Con frecuencia se generan controversias acerca de sí estas ganancias reflejan un riesgo financiero o económico.

Los que opinan que se afronta un riesgo financiero argumentan que esta ganancia está sujeta al pago del préstamo, un problema de liquidez y no necesariamente al riesgo del negocio. En cambio, los que opinan que se afronta un riesgo económico aducen que si el negocio va mal no existiría esta ganancia. Si estas ganancias estuvieran sujetas al riesgo financiero se descontarían a la tasa activa de mercado, si estuvieran afectadas al riesgo económico, se descontarían al COK. Además es importante anotar que una mayor tasa de descuento es beneficiosa para la GA y perjudicial para GEF.

Los tres enfoques de la GTI son aceptados. Este indicador mide las ganancias totales, tanto la que proviene del giro del negocio como la derivada del financiamiento.

Un caso simple ayudará a comprender mejor los conceptos en juego. Una empresa tiene un proyecto cuya inversión en activos fijos es de $\$ 100000$ un único flujo de ingreso de efectivo en el año uno, que no incluye ningún efecto por financiamiento, de \$150 000. La estructura financiera de la inversión es la siguiente: $\$ 60000$ con un préstamo subsidiado del $5 \%$ anual y $\$ 40000$ con aporte de capital propio. La tasa activa del mercado es $10 \%$ anual, el costo de oportunidad de capital (COK) 15\% anual y la tasa de impuesto fiscal $30 \%$. El proyecto tiene un horizonte de un año y los activos fijos se deprecian al $50 \%$ anual. Con esta información se tratará de analizar la bondad del proyecto. 
Cuadro 1 Flujo de caja(en Dólares)

\begin{tabular}{|c|c|c|c|}
\hline CONCEPTO & AÑO 0 & AÑO 1 & Liquidación \\
\hline Flujo de ingreso efectivo & & 150000 & \\
\hline Inversiòn & (100 000) & & 50000 \\
\hline Depreciación & & $(50000)$ & \\
\hline UAII=EBIT & & 100000 & \\
\hline Impuestos:30\%*100 000 & & $(30000)$ & \\
\hline Utilidad Neta & & 70000 & \\
\hline Más:Depreciación & & 50000 & \\
\hline Flujo de Caja Económico o Básico & $(100000)$ & 120000 & 50000 \\
\hline & \multicolumn{2}{|c|}{ Flujo de Financiamiento } & \\
\hline Préstamo & 60000 & & \\
\hline Amortización & & $(62000)$ & \\
\hline Intereses:0.05*60 000 & & $(3000)$ & \\
\hline Ahorro fiscal o Escudo Tributario: $0.3^{\star} 3000$ & & 900 & \\
\hline Flujo de Financiamiento Neto & 60000 & $(62100)$ & \\
\hline
\end{tabular}

VPNA = Valor Presente Neto Ajustado.

$\mathrm{GTI}=$ Ganancia Total del inversionista

VPN ganancia básica $=$ VPN ganancia económica

Primer Enfoque de I a GTI o Mét odo del Presupuesto de Capital

Para determinar la ganancia económica del proyecto o negocio, en forma sintética, previamente es necesario deducir los impuestos en el estado de resultados, y luego sumar la depreciación y la liquidación a la utilidad neta para así obtener el flujo económico que al descontarlo usando el COK sin apalancamiento, obtenemos la GEP (ver cuadro No 1).

GEP $=-100000+(120000+50000) / 1.15=\$ 47826.10$

Si se separa los dos tipos de ganancias por financiamiento, se tiene:

a. Ganancia por acceso al préstamo

Se debe tener en cuenta que el préstamo es por $\$ 60000$ y que se deberá devolver, después de un año, por concepto de intereses, $\$ 60000 \times 0.05$.

$$
\mathrm{GA}=60000-63000 / 1.10=\$ 2727.27
$$

b. Ganancia por escudo fiscal o escudo tributario:

$$
\mathrm{GAF}=0.3^{*} 3000 / 1.10=\$ 818.18
$$

Con los tres resultados anteriores puede hallarse la primera alternativa de la ganancia total del inversionista.

$\mathrm{GTI}=-100000+(120000+50000) / 1.10+(60000-63$ $000) / 1.10+900 / 1.10=\$ 51372$

$\mathrm{GTI}=\$ 51372$
Para visualizar mejor la GTI, es preciso elaborar el flujo de caja económico ( veáse Cuadro 1).

Por lo tanto, la ganancia económica es: GTI = VPNA

$\mathrm{GTI}=$ VPN ganancia económica + VPN ganancia por financiamiento

$\mathrm{GTI}=-100000+(120000+50000) / 1.15+60000-62$ $100 / 1.1=\$ 51372$, o alternativamente:

GTI =VPN ganancia económica + VPN ganancia por deuda + VP ganancia. fiscal

$\mathrm{GTI}=-100000+(120000+50000) / 1.10+60000-63000 /$ $1.10+900 / 1.10=\$ 51372$

Segundo Enfoque de I a GT

Se obtiene utilizando el WACC. Pero, primero es necesario convertir el COK en uno apalancado. El criterio del WACC se aplica al proyecto de inversión o plan de negocio cuando éste tiene similar nivel de riesgo y apalancamiento que la empresa

$$
\begin{aligned}
& \text { COKApal }=\text { COK }+(1-t)(C O K-i) D / C \\
& \text { COKApal }=0.15+(0.70)(0.15-0.05)(1.5)=25.5 \% \\
& \text { W A C C }=(1-t){ }^{*} i d\left(D /(C+D+P)+C O K^{*} C /\right. \\
& (C+D+P)+r^{\star} A p /(C+D+P)
\end{aligned}
$$

El último sumando sólo se tiene en cuenta cuando existe accionistas preferentes.

$$
\text { WACC }=0.05(1-0.3)(60 / 100)+0.255(40 / 100)+
$$
cero $=12.3 \%$ 
Cuadro 2. Flujodecajafinanciero(en Dólares)

\begin{tabular}{|c|c|c|c|}
\hline CONCEPTO & AÑO O & AÑO 1 & Liquidación \\
\hline Flujo de ingreso efectivo & & \begin{tabular}{|l|}
150000 \\
\end{tabular} & \\
\hline Inversiòn & (100 000) & & 50000 \\
\hline Depreciación & & $(50000)$ & \\
\hline UAII=EBIT & & 100000 & \\
\hline Intereses: $0.05^{\star} 60000$ & & $(3000)$ & \\
\hline UAI & & 97000 & \\
\hline Impuestos:30\%*97000 & & $(29100)$ & \\
\hline Utilidad Neta & & 67900 & \\
\hline Más: $\quad$ Depreciación & & 50000 & \\
\hline Intereses & & 3000 & \\
\hline Flujo de Caja Económico & (100 000) & 120900 & 50000 \\
\hline Más: & \multicolumn{2}{|c|}{ Flujo de Financiamiento } & \\
\hline Préstamo & \begin{tabular}{|r|}
60000 \\
\end{tabular} & & \\
\hline Amortización & & $(60000)$ & \\
\hline Intereses: $0.05^{\star} 60000$ & & (3000) & \\
\hline Ahorro fiscal: $0.30 * 3$ & & 900 & \\
\hline Flujo de caja financiero & $(40000)$ & 58800 & 50000 \\
\hline
\end{tabular}

Por lo tanto, aplicando el valor del WACC al flujo de caja económico o básico del Cuadro 1, se obtiene:

$$
\mathrm{GTI} 2=-\$ 100000+\$ 170000 / 1.18=\$ 51372
$$

Ter cer Enfoque de I a GT

Exige hallar un flujo de caja financiero el cual se descuenta con el COK apalancado, aunque la liquidación deberá descontarse con el WACC.

\section{$\mathrm{GTI}=-\$ 40000+\$ 58800 / 1.225+\$ 50000 / 1.123=$ $\$ 51376$}

De este modo, utilizando adecuadamente las tasas de descuento, se puede verificar que los 3 enfoques de la GTI ofrecen resultados iguales.

Otro caso sencillo, pero suponiendo flujos perpetuos, que puede ayudar aun más, a precisar los conceptos de tasa de descuento. Un proyecto empresarial, que inicialmente es financiado sólo con capital propio, tiene una inversión en activos fijos de $\$ 480$ 000, un flujo anual de ingresos por ventas en efectivo que no incluye ningún efecto por financiamiento de $\$ 600000$ y costos en efectivo de $74 \%$ sobre las ventas. Luego se decide tomar un préstamo en el mercado de capitales al $10 \%$ anual en una proporción fija de $25 \%$ sobre el valor presente (VP), lo cuál modifica la estructura financiera de la inversión. El costo de oportunidad de capital (COK) $20 \%$ anual y la tasa de impuesto marginal $40 \%$. El proyecto tiene un horizonte perpetuo. Se analizará la bondad del proyecto utilizando adecuadamente las diferentes tasas de descuento.
Primer criterio: el cual aplica el COK no apalancado al flujo de caja de caja económico para obtener el valor presente neto básico y aplica el costo del préstamo al flujo de financiamiento para obtener el valor presente del ahorro fiscal (ver Cuadro 3).

VP con deuda =VPNbásico+t.D/(C+D)VP con deuda $=468000+(0.40)(0.25) \mathrm{VP}$ con deuda $=0.90 \mathrm{VP}$ con deuda $=468000$

VP con deuda $=\$ 5200000$

Segundo criterio: el cual utiliza el costo promedio del capital(WACC). Entonces, previamente se calcula el WACC, para luego utilizarlo en descontar el flujo de caja sin apalancamiento o flujo de caja económico del Cuadro 3.

WACC $=(1-t)^{*} \mathrm{id}\left(\mathrm{D} /(\mathrm{C}+\mathrm{D}+\mathrm{P})+\mathrm{COK}^{*} \mathrm{C} /(\mathrm{C}+\mathrm{D}+\mathrm{P})+\mathrm{r}^{*} \mathrm{Ap} /\right.$ $(\mathrm{C}+\mathrm{D}+\mathrm{P})$

$W A C C=(1-0.4)^{*} 130 /(390+130)+0.2 * 390 /(390+130)+$ CeroWACC $=18 \%$ VPNA $=-480000+93600 / 0.18$ $=\$ 40000$

Tercer criterio: También llamado método del capital contable, el cual utiliza el COK apalancado. Para esto será necesario obtener el flujo de caja que considere el préstamo y sus intereses o flujo de caja financiero(ver Cuadro 4).

COK ajustado $=\mathrm{COK}+(1-\mathrm{t})(\mathrm{COK}-\mathrm{i}){ }^{*} \mathrm{D} /(\mathrm{D}+\mathrm{C})$

COK ajustado $=0.20+(1-0.40)(0.20-0.1)^{\star} 130 / 390$

COK ajustado $=22 \%$

VPNA $=-\$ 350000+\$ 85800 / 0.22=\$ 40000$ 
Cuadr o 3. Flujodecajaeconómico(en Dólares)

\begin{tabular}{|l|r|r|r|}
\hline CONCEPTO & AÑO O & AÑO 1 & Liquidación \\
\hline Flujo de ingreso efectivo & & 600000 & \\
\hline Inversiòn & $(480000)$ & & \\
\hline Costos en efectivo:74\% & & $(444000)$ & \\
\hline Ingreso de operación & & 156000 & \\
\hline Impuestos:40\% & & $(62400)$ & \\
\hline Flujo de Caja Económico & $(480000)$ & 93600 & \\
\hline Más: & \multicolumn{3}{|l|}{ Flujo de Financiamiento } \\
\hline Inver. ajust.al VP=520 000 & 130000 & & \\
\hline Préstamo=0.25(520 000) & & $(13000)$ & \\
\hline Intereses: 0.10*130 000 & & 5200 & \\
\hline Ahorro fiscal:0.40*13 000 & 130000 & $(7800)$ & \\
\hline Flujo de Financiamiento Neto &
\end{tabular}

Utilizando pertinentemente la tasa de descuento en este segundo caso, con flujos perpetuos, también se verifica que los tres enfoques de la GTI ofrecen resultados iguales

Errores en I a eval uación de inversiones En la revisión de numerosos planes de negocios suele encontrarse los siguientes errores:

Error 1: Sólo es importante la ganancia total del inversionista y la vida útil de los activos de un proyecto. Los proyectos más sólidos son aquellos que tienen capacidad de generar ganancias económicas más que ganancias por financiamiento. La vida útil de un proyecto no está en función de la vida útil de los activos (maquinaria o equipo) sino más bien de la capacidad del proyecto para generar valor. Por ende, el estudio de mercado y del entorno son cruciales para determinar la vida final del proyecto. La proyección de los ingresos en un proyecto de inversión sólo es la punta del iceberg del estudio de mercado

Error 2: No conviene continuar invirtiendo en un proyecto de inversión en marcha que tiene un VPN total negativo. Un proyecto de inversión que aún le falta desembolsar una parte de su inversión, y donde una parte de lo ya desembolsado se considera irrecuperable o costo hundido puede aún resultar conveniente continuar invirtiendo en él, a pesar de tener un VPN total negativo, sólo si el VPN de la parte que falta ejecutar del proyecto es mayor al VPN de la parte recuperable que ya ha sido ejecutada en él.

Error 3: Si no se puede medir del riesgo, éste no existe. Tal vez no se requerirá muchos datos históricos pero sí mucha imaginación para visualizar la realidad del futuro. Por ejemplo los proyectos enfrentan diferentes riesgos cuando enfrentan sucesos como los fenómenos del niño, las crisis financieras inter- nacionales o las crisis políticas; y aunque estos riesgos no puedan ser cuantificados con toda precisión, el riesgo existe

Error 4: Utilizar el costo promedio ponderado de capital (WACC) para descontar a los flujos financieros. EI WACC sólo se usa para descotar los flujos económicos. Con este error se está sobreestimando el verdadero valor del VPN total.

Error 5: Si la información disponible en el mercado de valores local es insuficiente, el riesgo dependerá de la estimación subjetiva del analista o de cuánto desea ganar el inversionista. El riesgo es un hecho objetivo. Es externo al proyecto. Es o no posible, que los expertos tengan mejor información que el mercado. Se suele confundir estimación subjetiva con interpretación de datos reales.

Error 6: Al evaluar algunos proyectos es válido aceptar que el costo de oportunidad de capital es el rendimiento alternativo que posee el inversor sin importar el riesgo que representa porque se acepta la creencia de que sólo importa el rendimiento alternativo per se y no el riesgo. Por ejemplo, se suele usar como tasa de descuento de un proyecto riesgoso la tasa de depósitos a plazo (de menor riesgo que el proyecto) debido a que el inversor tiene depositados sus recursos en estos activos. Con ello, se está sobreestimando el verdadero valor del proyecto, ya que no se exige una rentabilidad que tenga en cuenta el riesgo de pérdida. Por ello sumar o restar dos valores que reflejan distintos riesgos puede sobrevalorar o subvalorar riesgos.

Error 7: Es posible que el COK de una inversión sea inferior a la tasa activa de mercado. Una mayor tasa activa se explica por un mayor riesgo. Por ello, una tasa activa mayor que la tasa de descuento implica- 
Cuadro 4. Flujodecajafinanciero(en Dólares)

\begin{tabular}{|l|r|r|r|}
\hline CONCEPTO & AÑO O & AÑO 1 & Liquidación \\
\hline Flujo de ingreso efectivo & & 600000 & \\
\hline Inversiòn & $(480$ 000) & & \\
\hline Préstamo:25\%*520 000 & 130000 & & \\
\hline Costos en efectivo:74\% & & $(444000)$ & \\
\hline Intereses: $10 \%{ }^{*} 130000$ & & $(13000)$ & \\
\hline Ingreso de operac ant. Imp. & & 143000 & \\
\hline Impuestos:40\% & & $(57200)$ & \\
\hline Flujo de caja financiero & $\mathbf{( 3 5 0 ~ 0 0 0 0 )}$ & $\mathbf{8 5 8 0 0}$ & \\
\hline
\end{tabular}

ría que la empresa acepta un mayor riesgo que lo que representa su proyecto (ya que el riesgo del proyecto a evaluarse es igual al del COK) y esto es económicamente irracional. Si una empresa evalúa un proyecto cuyo COK es $20 \%$, que refleja razonablemente el riesgo del proyecto; ¿por qué la empresa aceptaría una tasa de préstamo de $25 \%$, que representa un riesgo mayor al de su proyecto?

Error 8: Si en el corto plazo, los rendimientos de la bolsa son negativos, el costo de oportunidad es cero. El horizonte de la estimación del rendimiento alternativo debe reflejar el rendimiento de largo plazo. El riesgo no tiene por qué ser igual en todos los periodos. Debe tratarse de identificar fases de riesgo en el proyecto. A menos que se estime que la bolsa desaparecerá, este rendimiento negativo no puede aceptarse.

Error 9: Cuando no hay manera de estimar adecuadamente los flujos de caja, cualquier estimación es válida. Se sabe que en la mayoría de Países, cada cierto tiempo, no mayor a cinco años, existen perturbaciones climáticas, económicas o financieras (fenómenos del Niño, crisis externas como en Argentina, Brasil, Indonesia, México, etc) que afectan a las empresas, y por ello son dudosos los proyectos con flujos constantes o crecientes a largo plazo. En un plan de negocios, elaborado en Argentina, se previó que los flujos económicos pasaban de $\$ 1$.2millones, en 1999, a \$3.6millones, en 2004, pero no previeron la crisis financiera de ese país que se produjo en el año 2002. Los mayores fracasos en los proyectos o negocios ocurren por un inadecuado análisis del entorno y del estudio de mercado.

Error 10: El mayor riesgo y el mayor horizonte son perjudiciales para un proyecto inversión. Este error se deriva de no entender los últimos avances de la teoría financiera que son explicadas por las opciones reales. Con las opciones reales el valor de todo proyecto o activo empresarial está dado por su valor actual más el valor de las opciones asociadas. El valor del primer componente se reduce al aumentar la incertidumbre (por efecto del mayor riesgo sobre la tasa de descuento y su impacto sobre el valor). Las opciones asociadas surgen de la flexibilidad que tiene el gestor de inversiones para responder a las condiciones cambiantes del entorno, aprovechando los estados favorables de la naturaleza para la empresa, o reduciendo pérdidas en los estados desfavorables. Hay 3 clases de opciones reales básicas: opción de espera, opción de crecimiento y opción de abandono. La opción de espera, refleja la flexibilidad que puede tener el gestor de inversiones en esperar a tomar una decisión de inversión hasta que la circunstancia sea aconsejable porque una inversión apresurada bajo incertidumbre, es similar a realizar una apuesta, y si eventualmente se puede esperar y ver como se desarrolla la incertidumbre, se podrá evitar invertir en malos escenarios. La opción de crecimiento, posibilita a la empresa capitalizar estados favorables de la naturaleza realizando inversiones adicionales si las cosas funcionan bien en una primera inversión (inversiones contingentes o condicionales en buenos estados de la naturaleza). La opción de abandono, permite al gestor de inversiones decidir abandonar o vender un proyecto que no tiene posibilidad de generar beneficios adicionales. Asimismo, cuando se presentan malos estados de la naturaleza y no hay posibilidad de reducir pérdidas o impedir que las mismas se incrementen mas el proyecto se vende por su valor alternativo (precio de ejercicio) que será mejor que su valor actual.

\section{EL CÁlCULO DE LA TASA DE DESCUENTO: NUEVA EVIDENCIA EMPÍRICA}

Dos enfoques dan respuesta a la estimación del riesgo: los que creen en el mercado y los que no creen en él.

\section{El Enfoque de Mercado}

Los que creen en el mercado, son aquellos que defienden el modelo de valoración de activos (Capital Asset Pricing Model CAPM). El CAPM es el único 


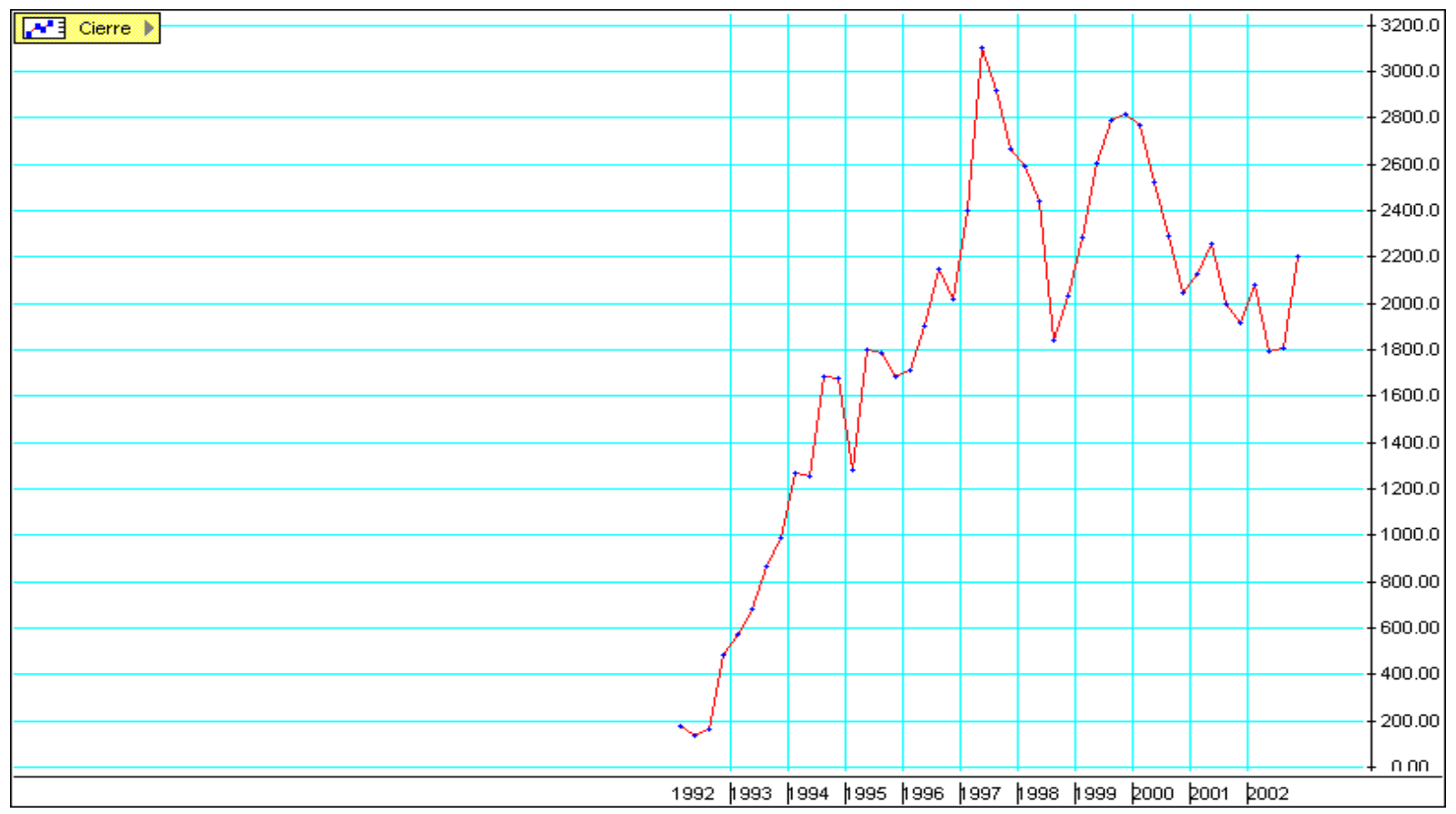

Figura 1 EvolucióntrimestraldelISBVL

Fuente: Economática, CONASEV

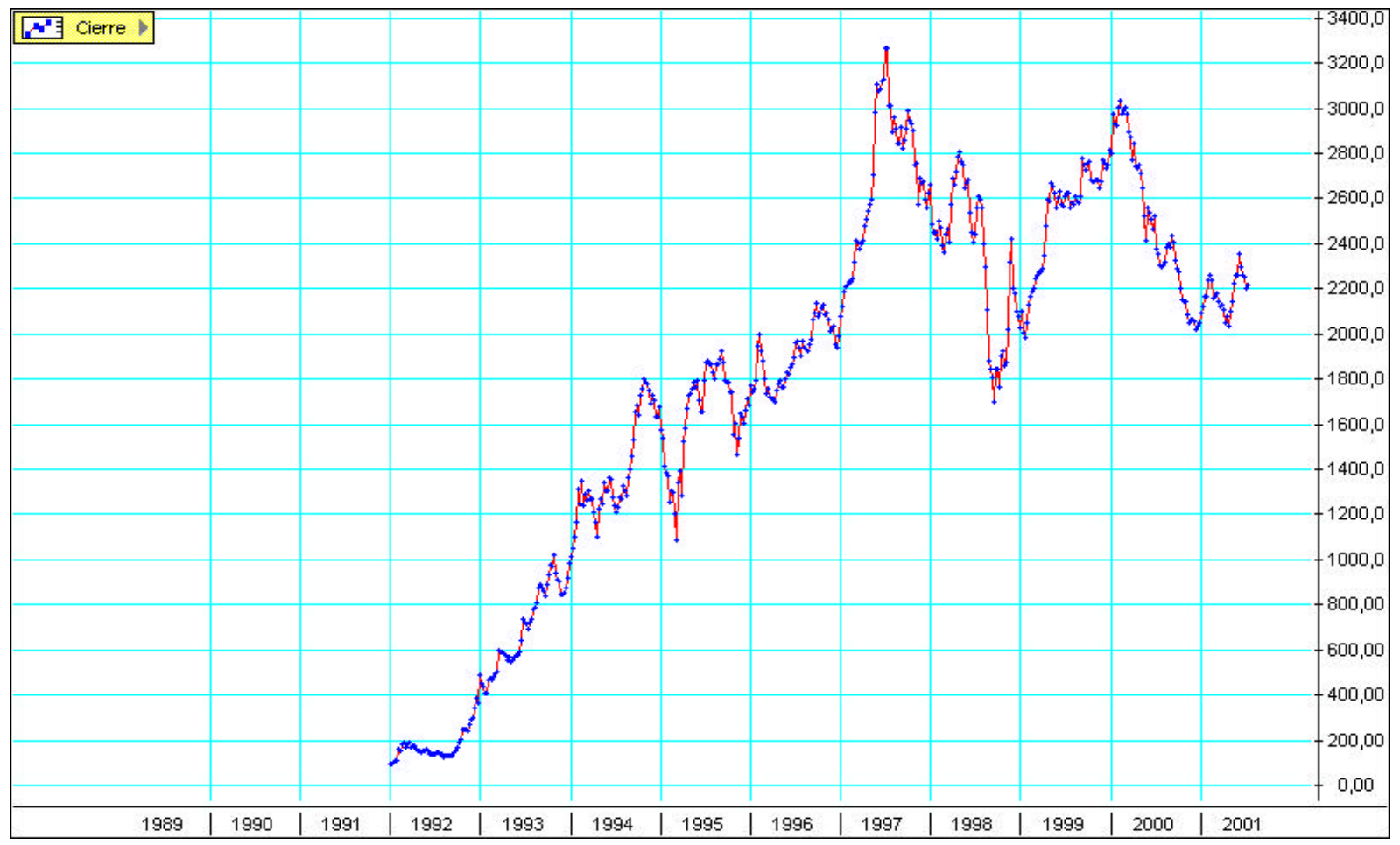

Figur a 2 EvoluciónmensualdelISBVL

Fuente: Economática, CONASEV 
criterio que incluye explícita y objetivamente el tema de riesgo.

En definitiva, una adecuada determinación de la tasa de descuento requiere que el mercado de valores tenga las siguientes características:

a. El mercado sea eficiente.

b. Que exista suficiente información pasada.

c. Que la información pasada prediga el futuro.

d. El riesgo del proyecto se mide por la desviación estándar de una acción con riesgo razonablemente igual al negocio evaluado.

Debe quedar claro que el mercado latinoamericano y en particular el mercado peruano no refleja plenamente estas características pero constituyen una hipótesis válida que es útil si se usa con buen criterio. A continuación se presentará, para el caso peruano, información válida para hallar el COK pertinente.

Para determinar el COK de la inversión es necesario disponer de una tasa libre de riesgo (Rf), un beta(B) y un rendimiento de mercado $(\mathrm{Rm})$, tal como ya se mencionó anteriormente, a través de la ecuación siguiente:

$$
\mathrm{COKi}=\mathrm{Rf}+\mathrm{b}(\mathrm{Rm}-\mathrm{Rf})
$$

A partir de esta relación se hallará los COKs válidos de las principales acciones (15) del índice selectivo de la Bolsa de Valores de Lima(ISBVL). Las otras acciones del índice general, no son representativas porque no todas son líquidas. Aún cuando se presentan dificultades, los dos primeros componentes no son difíciles de hallar. Se considera una buena tasa libre de riesgo a los bonos de tesoro americano (T-Bill)desde un año hasta 30 años que actualmente se encuentran en $8.4 \%$ y $5.8 \%$ por año respectivamente. Teóricamente, en un mercado global perfecto la Rf sería única. Los beta de las acciones pueden hallarse simplemente en una base de datos como Economática.

El Rm implica cierta dificultad de medición. Actualmente, según el Investment Portfolio Quarterly(winter 2004) el Rm válido para el Perú y la mayor parte de Latinoamérica, debe ser superior a $18 \%$ anual, debido a que el mercado de acciones en Latinoamérica se consideran más riesgosas que las del mercado norteamericano y general en los llamados países del norte. El rendimiento promedio de acciones ordinarias según el índice valores comenzaron a recuperarse en el año 2 003, después de la guerra energética global en el medio oriente. Así, al 30 de septiembre del 2003 el índice de retorno global compuesto S\&P/TSX llegó a 18.04\% en EUA, el S\&P500 gano en el mismo período $18.45 \%$, y el NASDAQ alcanzó 33.24\%. Pero en el largo plazo, sin tomar en cuenta la coyuntura de la guerra, ese rendimiento promedio es de aproximadamente $13 \%$. Volviendo al caso del Perú, en las Figuras 1 y 2 se pueden observar un período relativamente estable para el Perú, en donde se muestra el comportamiento del índice selectivo de la Bolsa de Valores de Lima (ISVBL). Se puede dividir el comportamiento del índice en cuatro períodos:

a. antes de 1992

b. entre 1992 y 1994

c. entre 1994 y 1997

d. después de 1997

Primero, se analiza qué períodos no son estables. Antes de 1992, la bolsa era muy pequeña, la capitalización bursátil era inferior a los $\$ 1,000$ millones, hoy supera los \$10 000 millones. Entre 1992 y 1994 el perú comienza a convertirse en un gran mercado emergente. Los precios de las acciones subieron coyunturalmente para situarse en los niveles internacionales, por ello tampoco reflejaban expectativas de rendimiento futuro. Después de 1996, se originó una recesión que desalentó la inversión tanto en el sector real como en la bolsa, debido a la crisis del Asia y las fuertes acusaciones de corrupción interna. Se puede ver que esta última etapa tampoco refleja un período estable.

Pero, existe un breve período entre el 1 de junio de 1994 y 31 de julio 1997, que puede considerarse estable. Según un estudio preliminar se concluye que este período se constituye en un mercado eficiente al menos en su hipótesis débil(los precios a ese período reflejan toda la información pasada).

En ese período la rentabilidad anual fue de $15.79 \%$ anual en dólares $(0.061715 \%$ diaria x 256 días). Con esto es posible determinar el COK para estas quince acciones del índice selectivo que se muestran en el Cuadro No. 5. Puede observarse que trece acciones ofrecen datos para hallar su tasa de descuento y dos no. El Banco Wiese Sudameris tiene un beta de 1, tres acciones superan este beta de 1 y nueve acciones se sitúan por debajo de este beta promedio. Además, puede observarse que, a excepción de Atacocha en los primeros tres años, los beta son razonablemente estables en el tiempo; y que aproximadamente las empresas clasificadoras de riesgos ordenan las acciones de menor a mayor riesgo de igual manera que los beta.

Los COKs ofrecen un mínimo de consistencia; oscilan entre un mínimo de $8 \%$ para Edegel (empresa de servicios públicos) y $30 \%$ de Atacocha (empresa minera que comercializa productos con precios volátiles). Si se cometiera el error de considerar la tasa 
Cuadr o 5. Tasade descuento de las acciones del ISBVL-Perú

\begin{tabular}{|l|c|c|c|c|}
\hline Empresa & Beta $^{\mathbf{1}}$ & COK (\%) & Rating $^{*}$ & Deuda/Patrim. $^{\mathbf{~}}$ \\
\hline Edegel S.A. C1 & 0.2 & 7.6 & $1 \mathrm{ra}$. & 0.5 \\
\hline Luz del Sur S.A. C1 & 0.3 & 8.7 & $1 \mathrm{ra}$. & 1.1 \\
\hline Casa Grande S.A. C1 & 0.4 & 9.7 & & 0.5 \\
\hline Banco Continental C1 & 0.4 & 9.7 & $1 \mathrm{ra}$. & 10.2 \\
\hline Buenaventura BC1 & 0.6 & 11.7 & $1 \mathrm{ra}$. & 0.1 \\
\hline Southern Perú C.C. C1 & 0.7 & 12.7 & $1 \mathrm{ra}$. & 0.8 \\
\hline Bco. Wiese Sudameris C & 0.8 & 13.8 & $2 \mathrm{da}$. & 8.7 \\
\hline Minsur I1 & 0.9 & 14.8 & & 0.2 \\
\hline UCP Backus \& Johnston I & 0.9 & 14.8 & $1 \mathrm{ra}$. & 0.6 \\
\hline Telefonica del Perú BC1 & 1 & 15.8 & $1 \mathrm{ra}$. & 1.6 \\
\hline Credicorp ADR & 1.6 & 21.9 & $1 \mathrm{ra}$. & 9.3 \\
\hline Volcan BC1 & 2 & 26 & $3 \mathrm{ra}$. & 0.9 \\
\hline Atacocha I1 & 2.4 & 30.1 & $3 \mathrm{ra}$. & 0.6 \\
\hline Milpo BC1 & & & & 0.4 \\
\hline Telefonica S.A. ADR & & & & \\
\hline
\end{tabular}

1 Al 17 de julio del 2001, precios en dólares de los últimos 36 meses, respecto al ISBVL.

2 A marzo del 2001.

* Según la clasificación más conservadora.

Fuente: Economática, D. Wong.

Cu ad r o 6. Betas calculados con información mensual de 36 meses (en \$) respecto al ISBVL-Perú

\begin{tabular}{|r|l|c|c|c|c|c|c|c|}
\cline { 2 - 9 } \multicolumn{1}{|l|}{ Empresa } & Jul-01 & Dic-00 & Dic-99 & Dic-98 & Dic-97 & Dic-96 & Dic-95 \\
\hline $\mathbf{2}$ & Atacocha I1 & 2.4 & 2.2 & 2.5 & 2.6 & 1.7 & 0.8 & 0.6 \\
\hline $\mathbf{3}$ & Bco. Wiese Sudameris C & 0.4 & 0.5 & 0.6 & 0.6 & 0.6 & 0.8 & \\
\hline $\mathbf{4}$ & Buenaventura BC1 & 0.6 & 0.8 & 0.6 & 0.8 & 0.6 & 0.9 & 0.7 \\
\hline $\mathbf{5}$ & Casa Grande S.A. C1 & 0.4 & 0.2 & & & & & \\
\hline $\mathbf{6}$ & Credicorp ADR & 1.6 & 1.3 & 1.2 & 1.2 & & & \\
\hline $\mathbf{7}$ & Edegel S.A. C1 & 0.2 & 0.4 & 0.5 & & & & \\
\hline $\mathbf{8}$ & Luz del Sur S.A. C1 & 0.3 & 0.4 & 0.4 & & & & \\
\hline $\mathbf{9}$ & Milpo BC1 & & & & & & & \\
\hline $\mathbf{1 0}$ & Minsur I1 & 0.9 & 0.9 & 0.8 & 0.8 & 0.8 & 0.9 & \\
\hline $\mathbf{1 1}$ & Southern Perú C.C. C1 & 0.7 & 0.7 & 0.7 & 0.6 & & & \\
\hline $\mathbf{1 2}$ & Telefonica del Perú BC1 & 1 & 1 & 0.9 & 0.8 & 1 & 1.1 & 1 \\
\hline $\mathbf{1 3}$ & Telefonica S.A. ADR & & & & & & & \\
\hline $\mathbf{1 4}$ & UCP Backus \& Johnston & 0.9 & 0.9 & 1 & 1 & 0.9 & 0.9 & 0.8 \\
\hline $\mathbf{1 5}$ & Volcan BC1 & 2 & 1.8 & & & & & \\
\hline
\end{tabular}


descuento como $8 \%$ y no 30\%, el máximo error de un proyecto con flujos económicos perpetuos sería:

$$
V P=(100000 / 0.08)-(100000 / 0.30)=\$ 916667
$$

Las tasas de descuento (\%) proyectadas son las siguientes:

$\begin{array}{ll}\text { Depósitos a plazo } & 3.0 \text { a } 5.0 \\ \text { Fondos mutuos de renta fija } & 5.0 \text { a } 7.0 \\ \text { Tasa activa promedio } & 13.0 \\ \text { Bolsa beta }=1 & 16.0 \\ \text { Bolsa beta }=2.4 & 30.0\end{array}$

En los proyectos de inversión peruanos los COKs deben situarse dentro de estos rangos. Obviamente es imposible determinar con certeza la exacta tasa de descuento de cada proyecto, pero es posible seguir con los criterios siguientes:

a. Los proyectos de inversión no constituyen activos de renta fija por lo que generalmente se situarán alrededor del rendimiento especulativo de beta $=$ 1. Cabe preguntarse ¿en dónde es preferible invertir, en el Banco Wiese o en un proyecto evaluado?

b. Salvo casos excepcionales, como las empresas de servicios públicos (Edegel, Telefónica, etc.), la tasa de descuento estará por encima de la tasa activa promedio de mercado.

c. Es imposible asegurar que un proyecto riesgoso (nuevo, o con demanda no comprobada, o ubicada en sector de gran competencia, etc.) tenga un COK de $30 \%$, pero tampoco es posible asegurar que sea $16 \%$ (beta $=1$ ). Por ello es indispensable establecer una simulación, entre ambas tasas, para observar la sensibilidad del proyecto a la tasa de descuento. Debe recordarse que un COK de $16 \%$ es la versión optimista(que genera un mayor VPN) frente a un COK igual a $30 \%$ (que genera un menor VPN).

d. En ocasiones, el COK podría ser superior a la tasa del $30 \%$.

e. Una mayor precisión para hallar la tasa de descuento exigiría la consulta a expertos en el negocio.

Enf oque de I os que no creen en el Mer cado Los fundamentalistas o los que no creen en el mercado afirman que, debido a que los precios en el mercado de capitales peruano están distorsionados el modelo del CAPM no es válido. Por ello se basan en valores emitidos por expertos en el sector y en valores contables.

Una gran ventaja en la opinión de expertos es que existen en mayor número que los sectores representados en la bolsa de valores. Sin embargo, sus opiniones normalmente no están disponibles.
Los valores contables tienen la ventaja, al igual que la opinión de expertos, de su mayor disponibilidad. Así por ejemplo, se afirma que un indicador imperfecto del costo de oportunidad es el rendimiento sobre la inversión (utilidad/capital propio). No obstante, estos indicadores no evidencian riesgo y por ello son muy dudosos.

\section{CONCLUSIONES}

No cabe duda la importancia que tiene la calidad de las decisiones de inversión para la buena marcha de la empresa y donde la determinación de la tasa de descuento genera muchas controversias, lo cual cobra mayor relevancia en los mercados emergentes, como en el caso de los mercados latinoamericanos. El analista o gestor de inversiones puede tomar puntos de referencia y rangos razonables entre los cuales podría fluctuar el valor de la tasa de descuento.

Son ciertas las innumerables críticas que se hacen a todos los métodos para calcularla. Sólo hay estimaciones gruesas. No hay cálculos exactos, sobre todo en un mercado incompleto como el peruano donde muy pocas empresas cotizan en la bolsa de valores y las series estadísticas disponibles son cortas, volátiles y poco confiables, lo cual conduce a errores estándares amplios. Además, los mercados son pequeños y dominados por muy pocas empresas.

En el caso peruano cuando se calcula la correlación de una empresa con el mercado, en realidad se la está correlacionando con las empresas dominantes. A pesar de los escollos existentes, es necesario un esfuerzo que implique lógica y consistencia. Por eso la evaluación de inversiones no es la aplicación automática de fórmulas, sino un ejercicio de razonamiento dinámico.

En la práctica, como lo perfecto es enemigo de lo bueno, hay que buscar un método imperfecto que presente las menores limitaciones; y probablemente éste siga siendo el CAPM porque es el único que estima objetivamente los factores más importantes de un proyecto de inversión: la rentabilidad y el riesgo, el cual toma como referencia los mercados maduros de los países desarrollados.

En los mercados emergentes latinoamericanos es posible utilizar el modelo CAPM para obtener la prima de riesgo del capital propio, tomando como referencia la prima básica del mercado de los EUA más la prima por el riesgo país. Para ello esa necesario medir el riesgo país y transformar ese riesgo país en una prima por riesgo país. Y luego evaluar cómo esas empresas están expuestas al riesgo país. 


\section{BIBLIOGRAFÍA}

1. Bolsa de Valores de Lima. (2004). Indicadores Bursátiles: 1992-2003. En: http://www.bvl.com.pe/

2. Brealey A., Richard. (1993). Principios de Finanzas Corporativas. 2da. Edición. Mc Graw-Hill, México.

3. Enders, Walter. (1995). Applied Econometric Time Series. John Wiley and Sons, Inc. USA.

4. Engle, Robert F.; David, Lilien y Russell, Robins (1987). Estimating Time Varying Risk Premia in the Term Structure, The Arch-m Model. Ecomometrica 55, marzo, pp. 391-407. En: http:// www.jstor.org/journals/00129682.html

5. Fama, E. F. (1971). Risk Return and Equilibrium. Journal of Political Economy, 79, enero y febrero, pp. 30-55. En: http://www.journals.uchicago.edu/ JPE/home.html

6. , y J. Mac Beth. (1973). Risk, Return and Equilibrium. Empirical Test. Journal of Political Economy, 81, mayo-junio, pp. 607-636. En: http:/ /www.journals.uchicago.edu/JPE/home.html

7. Luehman, Timothy. (1998). Investment Opportunities as Real Options. HBR, julio-agosto 1998. En: http:/ /harvardbusinessonline.hbsp.harvard.edu/b02/en/ hbr/hbr_home.jhtml

8. Ross, Westerfield y Jaffe. (1997). Finanzas Corporativas. McGraw Hill, México.

9. Weston, Fred, y Thomas E. Copeland. (1992). Finanzas en Administración. 3ra. Edición. Mc GrawHill, México. 